\title{
Evaluación de competencias con metodologías de aprendizaje basado en proyectos
}

\author{
Iñigo Cuiñas ${ }^{\mathrm{a}}$, Perfecto Mariño-Espiñeira ${ }^{\mathrm{b}}$, Manuel Fernández-Iglesias ${ }^{\mathrm{c}}$, Manuel \\ Caeiro $^{c}$, Enrique Costa-Montenegro ${ }^{c}$ y Francisco Díaz-Otero ${ }^{a}$
}

${ }^{a}$ Dept. Teoría do Sinal e Comunicacións, Universidade de Vigo (inhigo@uvigo.es; fjdiaz@com.uvigo.es), ${ }^{b}$ Dpto. Tecnoloxía Electrónica, Universidade de Vigo (pmarino@uvigo.es) y ${ }^{\mathrm{c}}$ Dpto. Enxeñaría Telemática, Universidade de Vigo (manolo@uvigo.es; mcaeiro@det.uvigo.es; kike@det.uvigo.es)

\begin{abstract}
After two years teaching a course on project based learning following the Design Thinking methodology within the Master in Telecommunication Engineering at the University of Vigo, the authors present their experience. With that aim, the course is introduced, taking especial care in presenting the skills that will be developed and evaluated following Design Thinking dynamics. Then, we explain the process of team construction, its development and the evaluation procedure, in which both lecturers and students take part following rubrics previously known and published at the beginning of the course. Besides, we analyse the results of marking the works developed by the teams, considering both lecturers and peers evaluations, as well as the students satisfaction for being involved in such a experience.
\end{abstract}

Keywords: transversal competences, Design Thinking, peer evaluation, team working, rubric.

\footnotetext{
Resumen

Tras dos cursos impartiendo una asignatura de aprendizaje basado en proyectos según la metodología Design Thinking en el Máster en Ingeniería de Telecomunicación de la Universidad de Vigo, los autores presentan su experiencia. Para ello se introduce la asignatura, con especial énfasis en la presentación de las competencias que se desarrollan y evalúan según dinámicas de Design Thinking. Posteriormente se expone el proceso de definición de los grupos, su desarrollo y el procedimiento de evaluación, en el que intervienen tanto docentes como alumnos siguiendo unas rúbricas conocidas y publicadas al principio del curso. También se analizan los resultados de la evaluación de los trabajos paralelizando las valoraciones de profesores y alumnos, asi como la evaluación de la satisfacción que muestran los alumnos al participar en una experiencia de este tipo.
}

Palabras clave: competencias transversales, Design Thinking, evaluación por pares, trabajo en equipo, rúbrica. 


\section{Introducción}

La titulación de Máster en Ingeniería de Telecomunicación, que habilita para la profesión regulada homónima según la orden CIN/355/2009 (Orden CIN, 2009), incluye una serie de competencias que todos los alumnos deben adquirir, relacionadas con aspectos sociales de la Ingeniería. En la definición del programa formativo de la Escuela de Ingeniería de Telecomunicación, estas competencias están asignadas a la asignatura "La Ingeniería de Telecomunicación en la Sociedad de la Información" (Caeiro, 2015). La asignatura busca sacar al alumno de los conceptos más técnicos de Ingeniería de Telecomunicación y centrarlo en la sociedad en la que vive: se pretende que tome conciencia de que la actividad del ingeniero no es un hecho aislado sino que transforma al mundo (a pequeña y a gran escala). Esto lleva a dos ideas fundamentales:

- La sociedad, las personas que la conforman, tienen problemas que pueden ser resueltos por los ingenieros: la función de la Ingeniería es resolver o mitigar problemas de la sociedad en la que se enmarca, no crearlos. El cómo se han resuelto situaciones en el pasado puede ayudar a encarar problemas en el futuro (historia orientada a la acción futura, no a la contemplación del pasado).

- Las actividades ingenieriles tienen influencia directa en la propia sociedad, en cómo viven o en cómo se relacionan las personas. De hecho, los grandes cambios de las últimas décadas han estado protagonizados directamente por aportaciones del ámbito de la Ingeniería de Telecomunicación. Esta influencia debe ir acompañada de una toma de conciencia de la responsabilidad ética.

Con esta definición de principios, la asignatura consiste en una parte de aula, centrada en conferencias sobre las atribuciones profesionales de la Ingeniería de Telecomunicación y los ámbitos de actuación profesional de sus titulados, en la que se cuenta con egresados de la Escuela explicando su desarrollo laboral. La asignatura también consta de un módulo que llamamos "En una Sociedad Multidisciplinar", en el que se propone a los alumnos la resolución de problemas o situaciones de la sociedad en la que vivimos, no necesariamente relacionados con la Ingeniería de Telecomunicación, para que los alumnos comprendan su implicación en múltiples ámbitos de la sociedad y cómo pueden influir en ella con soluciones planteadas desde sus competencias y habilidades ingenieriles. No se trata de fabricar o programar una solución, sino de buscar una propuesta que sea factible, ahora o en un futuro con tecnología más desarrollada, y que sea aceptable socialmente.

El "pensamiento para el diseño" o Design Thinking (DT) (Johansson-Sköldberg, 2013; Owen, 2007) es una metodología iterativa para el desarrollo de proyectos que se basa en aplicar el enfoque y las técnicas habituales del mundo del diseño y el marketing a otras disciplinas. En concreto, DT propugna un enfoque novedoso para el desarrollo de proyectos organizado en una serie de etapas, que se identifican habitualmente como empatía, definición, generación de ideas, prototipado y experimentación. Esta metodología fue desarrollada en el Institute of Design at Standford y, además de convertirse en una metodología académica orientada al aprendizaje basado en proyectos, ha sido aplicada por numerosas empresas de éxito en todo tipo de sectores, desde el marketing a la Ingeniería.

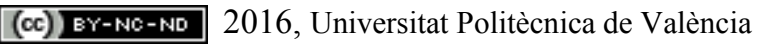


Mediante la aplicación de una aproximación centrada en las personas y basada en la realización de pruebas iterativas con prototipos, se alcanzan soluciones innovadoras y conectadas con las necesidades reales de los usuarios.

Esta comunicación se centra en la parte de la asignatura orientada a la realización de proyectos: tratamos de describir el proceso de construcción de los grupos, su desarrollo y la evaluación de los proyectos que realizan. Para ello, la segunda sección describe los objetivos de la propuesta, mostrando qué competencias se pretenden desarrollar y evaluar con la actividad planteada. La tercera sección está constituida por la descripción de las actividades de construcción de los grupos, el desarrollo de la actividad y el proceso de evaluación. En la cuarta sección evaluamos los resultados, tanto cuantitativos como cualitativos de la experiencia. Finalmente, la quinta sección recoge las conclusiones de la misma.

\section{Objetivos}

El objetivo de la propuesta es definir el procedimiento más adecuado para construir grupos, desarrollar los proyectos y evaluarlos, dentro de una dinámica DT, de modo que se cubran las competencias correspondientes de la ficha de la asignatura "La Ingeniería de Telecomunicación en la Sociedad de la Información", enunciadas a continuación tal y como están escritas en la memoria del programa formativo:

- Competencia básica 1: Que los estudiantes sean capaces de integrar conocimientos y enfrentarse a la complejidad de formular juicios a partir de una información que, siendo incompleta o limitada, incluya reflexiones sobre las responsabilidades sociales y éticas vinculadas a la aplicación de sus conocimientos y juicios.

- Competencia específica 1: Capacidad para la integración de tecnologías y sistemas propios de la Ingeniería de Telecomunicación, con carácter generalista, y en contextos más amplios y multidisciplinares como por ejemplo en bioingeniería, conversión fotovoltaica, nanotecnología, telemedicina.

- Competencia específica 2: Capacidad para comprender la responsabilidad ética y la deontología profesional de la actividad de la profesión de Ingeniero de Telecomunicación.

- Competencia transversal 1: Tomar conciencia de la necesidad de una formación y mejora continua de calidad, desarrollando valores propios de la dinámica del pensamiento científico, mostrando una actitud flexible, abierta y ética ante opiniones o situaciones diversas, en particular en materia de no discriminación por sexo, raza o religión, respeto a los derechos fundamentales, accesibilidad, etc.

Con estas competencias en mente, comenzamos por sumergir a los alumnos en un proceso lo más real posible de selección de personal a la hora de constituir grupos con unos perfiles psicológicos predeterminados. A continuación, disponen de doce semanas para desarrollar su proyecto siguiendo una adaptación de la metodología DT, asesorados por un profesor.

(cc) EY-NC-ND 2016, Universitat Politècnica de València

Congreso In-Red (2016) 
Finalmente, presentan sus resultados a la clase y a los profesores de la asignatura, que los evalúan usando las rúbricas de profesor y de alumno.

\section{Desarrollo de la innovación}

En esta sección se describen los tres procesos principales del desarrollo del módulo "En una Sociedad Multidisciplinar", basado en una dinámica de aprendizaje por proyectos: la selección del personal, esto es, la construcción de los grupos; el desarrollo de la actividad; y la evaluación.

Previamente a la actividad grupal en sí misma, los estudiantes reciben una formación inicial sobre el uso de la metodología DT para hacer frente a un desafío dado. Durante esta formación, se hace especial énfasis en la indicación clara de las cinco fases del proceso: la empatía/descubrimiento, definición, ideación, creación de prototipos y pruebas (IDEO 2012).

- Fase de empatía/descubrimiento:

- Se trata de analizar y comprender un desafío dado, asociar "qué buscar/descubrir" con los posibles interesados, diseñar un mapa de interesados.

- Mediante una salida de campo, se propone a los alumnos sumergirse en un lugar inspirador y entrevistar a las partes interesadas, sobre la base de un esquema de entrevista articulado en torno a las preguntas abiertas (aprendiendo, de paso, el arte de preguntar).

- Fase de definición/identificación del problema:

- Sintetizar los hallazgos utilizando herramientas visuales tales como el mapa de empatía, el mapa de afinidad, la matriz de relaciones, el mapa conceptual, con el fin de encontrar percepciones o intuiciones y formular el llamado Punto de vista (POV).

- Fase de ideación:

○ Definir preguntas "¿cómo podemos...?" basadas en POV para tener un enfoque para la sesión de ideación.

- Lluvia de ideas con diferentes puntos de enfoque ("Sí, y también..."): ideas, ideas limitadas, ideas basadas en analogías.

- Lluvia de ideas para seleccionar las mejores: votando, mapas de comparación $2 \times 2$, etc.

- Fases de prototipado y pruebas:

- Crear prototipos de baja fidelidad mediante el dibujo, maquetas o haciendo pequeñas manualidades.

- Taller de Diseño: pensar en la forma de presentar el prototipo, que sea probado por el usuario y obtener retroalimentación.

- Fase de análisis: informe de retroalimentación (relación entre el POV y la solución obtenida) y pensar donde volver a iterar el proceso de DT para llegar a un resultado más preciso.

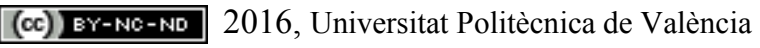




\subsection{Selección del personal}

La filosofía de formación de grupos de trabajo se establece como si constituyesen un grupo de diseño de la división de I+D de una Empresa de ingeniería (Liedtka, 2011), cuyo proyecto han de presentar al supervisor para que sea aceptado por la Empresa. En consecuencia los estudiantes del módulo cubren una encuesta sencilla que indique su especialidad y perfil psicológico, para que el grupo sea lo más multidisciplinar posible (Catmull, 2015) dentro del conjunto de matriculados. Así se intenta una aproximación a las prácticas habituales de las empresas reales en la selección de su personal de I+D.

El perfil psicológico está basado en la dicotomía extrovertido/introvertido de (Cain, 2012, pág. 32) para extraer líderes provisionales que puedan ser cambiados durante las sesiones de control, con el objetivo de cohesionar al grupo y aumentar la motivación de cada uno de sus integrantes (Kahneman, 2011). Además, se intenta que haya un equilibrio de género en cada grupo.

\subsection{Desarrollo de la actividad}

El control de las sesiones presenciales es realizado por el profesor supervisor bajo la metodología descrita en los párrafos siguientes.

\subsubsection{Fundamentos}

Se parte de un objetivo común: la realización de un sistema siguiendo la metodología DT mediante trabajo en equipo. El profesor supervisor del grupo nombra un investigador líder, que será el encargado del seguimiento y control de las tareas conjuntas del grupo.

En cada equipo de trabajo se adoptan responsabilidades compartidas, bajo un líder rotativo del grupo de investigadores entre sesiones de control. Entre cada sesión de evaluación, con la asistencia del profesor supervisor, cada grupo de alumnos desarrolla algunas de las fases de la metodología DT, según la formación inicial.

En cada sesión de evaluación, el líder de turno presenta el trabajo de cada investigador, y se encarga del cumplimiento de las tareas correspondientes de cada uno hasta esa sesión. El profesor supervisor recibirá un informe escrito, realizará preguntas a todos y cada uno de los miembros del equipo sobre el trabajo realizado, y señalará tareas para la siguiente sesión.

El objetivo de esta metodología es fomentar la responsabilidad y coherencia en la realización de las tareas para potenciar las capacidades del grupo.

\subsubsection{Sesiones de evaluación de informes con el supervisor}

(cc) EY-NC-ND 2016, Universitat Politècnica de València

Congreso In-Red (2016) 
A lo largo de la asignatura se desarrollan cinco sesiones presenciales con el profesor supervisor, cuyos objetivos y contenidos se muestran a continuación:

1. Elección del sistema a diseñar, nombramiento del líder, discusión y entregable (informe) de la siguiente sesión (índice). En esta primera sesión se determinan las líneas generales de lo que va a ser el trabajo: a quién va dirigido, qué se pretende resolver, etc. Esta sesión se corresponde con el principio de la fase de empatía o descubrimiento del problema.

2. Definición o identificación del problema. El equipo llega a esta reunión con el problema definido. Entregan un informe escrito en papel, que es presentado, evaluado y discutido, de modo que se define el problema en un nuevo informe. Nombramiento del nuevo líder hasta la próxima sesión.

3. Tras el trabajo de ideación en equipo, se realiza una sesión similar a la anterior, en la que el profesor supervisor ayuda al equipo a formalizar la idea de la solución que van a aportar.

4. Una vez realizada la primera versión del prototipo, se entrega el resultado final impreso, y se define una presentación de resumen del Sistema en Power Point, animaciones, vídeos o el propio prototipo. El equipo y su supervisor prueban la solución aportada y determinan qué es lo mejorable y en qué dirección van a trabajar para completar la tarea.

5. Con una versión refinada del sistema, en esta sesión se realiza una tarea similar a la anterior, pero ya centrada en la presentación final del sistema diseñado con participación de todos los investigadores del equipo. Es la reunión previa a la presentación de su resultado al grupo de toda la asignatura.

El objetivo de estas sesiones es depurar la realización de las tareas del grupo paso a paso para lograr un resultado de alta calidad en el diseño del sistema.

En algunos casos los informes y la presentación de los resultados se realizó a través de una Wiki. Utilizando el sistema wikispaces, los alumnos fueron documentando a lo largo de las distintas sesiones el trabajo realizado. Se trata de una especie de diario del proyecto en el que los alumnos documentan sus progresos y resultados. Un ejemplo del resultado puede verse en la Figura 1. De esta forma el profesor responsable del desarrollo del proyecto puede seguir fácilmente el trabajo de los alumnos y al mismo tiempo se va generando una Web que puede ser utilizada para la presentación del proyecto más allá del contexto académico. De forma particular, esta herramienta también se ha demostrado muy útil para permitir que los otros profesores de la asignatura que no se ocupan de la supervisión directa del grupo puedan hacer un seguimiento del trabajo realizado.

\subsection{Evaluación}

El proceso de evaluación se realiza mediante un método mixto: la mitad de la calificación la otorgan los compañeros de asignatura (que forman los otros equipos), según un procedimiento de evaluación por pares, y la otra mitad los profesores de la asignatura: tanto los que coordinan los proyectos como los que imparten los módulos de aula. Como la nota

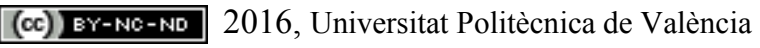


de los proyectos pesa un $30 \%$ de la nota de la asignatura, son los compañeros los que proporcionan un $15 \%$ y los profesores el otro $15 \%$.

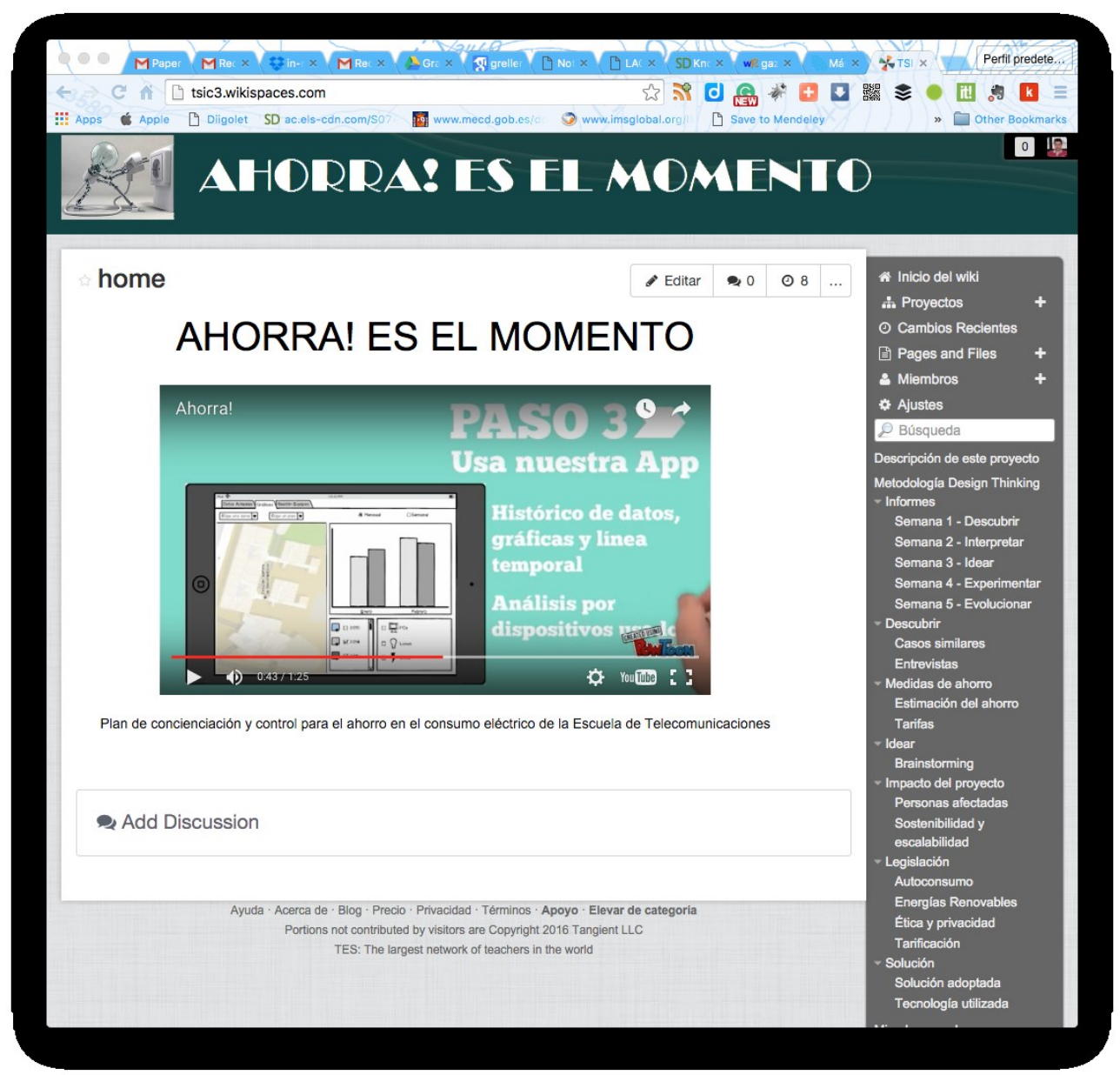

Figura 1. Página principla de la Wiki de uno de los grupos de trabajo. En la parte derecha puede verse las secciones correspondientes de la wiki, con indicaciones de cada una de las fases y las entregas realizadas.

La evaluación por parte de los alumnos se apoya en la rúbrica que se muestra en la tabla 1. En ella, se pide a los alumnos que observan la presentación que evalúen cada uno de los epígrafes que contiene entre 0 y 3 puntos, de modo que la suma total puede alcanzar, como máximo, 15 puntos. El uso de la rúbrica ayuda a guiar a los alumnos que evalúan por pares para que se fijen en las competencias correspondientes a la tarea y no en que la presentación de los resultados fuera más o menos divertida o agradable.

Tabla 1. Rúbrica de evaluación por parte de los alumnos (compañeros de los evaluados)

\begin{tabular}{|c|c|}
\hline Epígrafe & Evaluación \\
\hline
\end{tabular}

(c)) EY-NC-ND 2016, Universitat Politècnica de València

Congreso In-Red (2016) 


\begin{tabular}{|c|c|c|c|c|c|}
\hline & & 0 & 1 & 2 & 3 \\
\hline 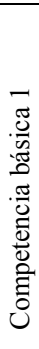 & $\begin{array}{l}\text { El trabajo muestra } \\
\text { capacidad para } \\
\text { enfrentarse a } \\
\text { problemas } \\
\text { complejos y para } \\
\text { emitir juicios a } \\
\text { partir de una } \\
\text { información inicial } \\
\text { parcial o limitada }\end{array}$ & $\begin{array}{l}\text { El grupo no ha } \\
\text { sido capaz de } \\
\text { presentar una } \\
\text { solución } \\
\text { completa y } \\
\text { justificarla } \\
\text { razonablemente }\end{array}$ & $\begin{array}{l}\text { El grupo presenta } \\
\text { una solución } \\
\text { parcial y } \\
\text { deficientemente } \\
\text { justificada }\end{array}$ & $\begin{array}{l}\text { La solución } \\
\text { resuelve un } \\
\text { problema } \\
\text { complejo, pero su } \\
\text { razonamiento no } \\
\text { es sólido }\end{array}$ & $\begin{array}{l}\text { El trabajo } \\
\text { presenta una } \\
\text { solución a un } \\
\text { problema } \\
\text { complejo, } \\
\text { justificada de } \\
\text { modo razonable }\end{array}$ \\
\hline 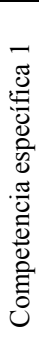 & $\begin{array}{l}\text { El trabajo muestra } \\
\text { integración de } \\
\text { conocimientos } \\
\text { previos de } \\
\text { Ingeniería de } \\
\text { Telecomunicación } \\
\text { en un contexto } \\
\text { amplio y } \\
\text { multidisciplinar. }\end{array}$ & $\begin{array}{l}\text { La solución } \\
\text { formulada } \\
\text { podría haberla } \\
\text { diseñado un } \\
\text { profesional de } \\
\text { cualquier otro } \\
\text { ámbito }\end{array}$ & $\begin{array}{l}\text { En la solución, el } \\
\text { grupo sólo ha } \\
\text { usado } \\
\text { parcialmente } \\
\text { alguna habilidad } \\
\text { propia de la } \\
\text { Ingeniería de } \\
\text { Telecomunicación }\end{array}$ & $\begin{array}{l}\text { La solución } \\
\text { permite } \\
\text { identificar } \\
\text { habilidades de la } \\
\text { Ingeniería de } \\
\text { Telecomunicación } \\
\text { aplicadas en otro } \\
\text { ámbito }\end{array}$ & $\begin{array}{l}\text { El grupo ha } \\
\text { aplicado } \\
\text { múltiples } \\
\text { conocimientos } \\
\text { previos para } \\
\text { buscar una } \\
\text { solución en un } \\
\text { ámbito } \\
\text { multidisciplinar }\end{array}$ \\
\hline 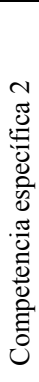 & $\begin{array}{l}\text { Capacidad para } \\
\text { comprender la } \\
\text { responsabilidad } \\
\text { ética y la } \\
\text { deontología } \\
\text { profesional de la } \\
\text { actividad de la } \\
\text { profesión de } \\
\text { Ingeniero de } \\
\text { Telecomunicación }\end{array}$ & $\begin{array}{l}\text { El grupo ignora } \\
\text { o viola los } \\
\text { principios } \\
\text { deontológicos } \\
\text { de la ingeniería } \\
\text { de teleco. }\end{array}$ & $\begin{array}{l}\text { Un debate } \\
\text { profundo podría } \\
\text { hacer aflorar } \\
\text { algún conflicto } \\
\text { ético o } \\
\text { deontológico. }\end{array}$ & $\begin{array}{l}\text { La solución es } \\
\text { compatible con } \\
\text { los principios } \\
\text { éticos y } \\
\text { deontológicos, } \\
\text { incluso tras su } \\
\text { debate. }\end{array}$ & $\begin{array}{l}\text { Los principios } \\
\text { éticos y } \\
\text { deontológicos se } \\
\text { tuvieron en } \\
\text { cuenta } \\
\text { explicitamente } \\
\text { en el diseño de la } \\
\text { solución. }\end{array}$ \\
\hline 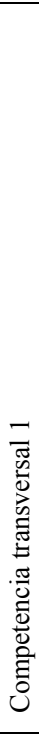 & $\begin{array}{l}\text { Conciencia de la } \\
\text { necesidad de una } \\
\text { formación y mejora } \\
\text { continua de } \\
\text { calidad, } \\
\text { desarrollando } \\
\text { valores propios de } \\
\text { la dinámica del } \\
\text { pensamiento } \\
\text { científico, con } \\
\text { actitud flexible, } \\
\text { abierta y ética ante } \\
\text { la diversidad (p. ej. } \\
\text { No discriminación } \\
\text { por sexo, raza o } \\
\text { religión, respeto a } \\
\text { los derechos } \\
\text { fundamentales, } \\
\text { accesibilidad, etc. }\end{array}$ & $\begin{array}{l}\text { Es evidente que } \\
\text { en el diseño de } \\
\text { la solución no } \\
\text { se consideraron } \\
\text { aspectos } \\
\text { relacionados } \\
\text { con el } \\
\text { aseguramiento } \\
\text { de la calidado } \\
\text { la sensibilidad } \\
\text { ante la } \\
\text { diversidad. }\end{array}$ & $\begin{array}{l}\text { La solución es } \\
\text { neutra con } \\
\text { respecto a la } \\
\text { diversidad en } \\
\text { general y } \\
\text { garantiza la no } \\
\text { discriminación, } \\
\text { aunque adolece } \\
\text { de algunas } \\
\text { limitaciones en } \\
\text { cuanto a } \\
\text { accesibilidad. }\end{array}$ & $\begin{array}{l}\text { La solución es } \\
\text { totalmente } \\
\text { accesible de } \\
\text { acuerdo con } \\
\text { criterios } \\
\text { razonables, y } \\
\text { respeta la } \\
\text { diversidad en } \\
\text { todas sus } \\
\text { vertientes. }\end{array}$ & $\begin{array}{l}\text { Una actitud } \\
\text { flexible y abierta } \\
\text { frente a la } \\
\text { diversidad en } \\
\text { todos sus } \\
\text { aspectos forma } \\
\text { parte del diseño } \\
\text { de la solución, } \\
\text { desde el momento } \\
\text { de su concepción } \\
\text { inicial y a lo } \\
\text { largo de todo el } \\
\text { proceso }\end{array}$ \\
\hline
\end{tabular}

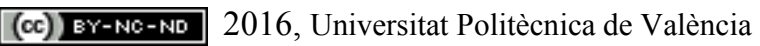




\begin{tabular}{|c|c|c|c|c|c|}
\hline & \multirow{2}{*}{ Epígrafe } & \multicolumn{4}{|c|}{ Evaluación } \\
\hline & & 0 & 1 & 2 & 3 \\
\hline 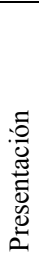 & $\begin{array}{l}\text { La presentación del } \\
\text { resultado muestra } \\
\text { una solución } \\
\text { atractiva, creativa y } \\
\text { razonable a un } \\
\text { problema complejo. }\end{array}$ & $\begin{array}{l}\text { La presentación } \\
\text { no muestra una } \\
\text { solución clara } \\
\text { al problema } \\
\text { identificado }\end{array}$ & $\begin{array}{l}\text { La solución es } \\
\text { razonable, pero } \\
\text { poco creativa. } \\
\text { Además su } \\
\text { presentación no } \\
\text { es atractiva }\end{array}$ & $\begin{array}{l}\text { La solución es } \\
\text { razonable, pero } \\
\text { poco creativa, } \\
\text { pero su } \\
\text { presentación es } \\
\text { atractiva }\end{array}$ & $\begin{array}{l}\text { La solución es } \\
\text { reflejo de } \\
\text { creatividad y } \\
\text { maduración de } \\
\text { ideas y su } \\
\text { presentación } \\
\text { resulta atractiva }\end{array}$ \\
\hline
\end{tabular}

Por su parte, la evaluación que realizan los profesores se basa en la rúbrica de la tabla 2. Como en el caso anterior, los profesores evalúan cinco epígrafes, diferentes a los que observan los alumnos, puntuando cada uno de 0 a 3 , hasta un máximo de 15 , que se correspondería con el $15 \%$ de la nota de la asignatura.

Tabla 2. Rúbrica de evaluación por parte de los profesores

\begin{tabular}{|c|c|c|c|c|c|}
\hline \multirow{2}{*}{\multicolumn{2}{|c|}{ Epígrafe }} & \multicolumn{4}{|c|}{ Evaluación } \\
\hline & & 0 & 1 & 2 & 3 \\
\hline 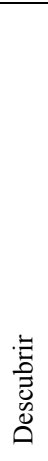 & $\begin{array}{l}\text { Los miembros del } \\
\text { grupo han sido } \\
\text { capaces de } \\
\text { recopilar } \\
\text { información } \\
\text { relacionada con el } \\
\text { problema } \\
\text { propuesto a partir } \\
\text { de diferentes } \\
\text { fuentes. Todos } \\
\text { son partícipes de } \\
\text { este proceso. }\end{array}$ & $\begin{array}{l}\text { La búsqueda de } \\
\text { información ha } \\
\text { sido escasa y } \\
\text { con poca } \\
\text { iniciativa }\end{array}$ & $\begin{array}{l}\text { Al menos parte } \\
\text { del grupo ha } \\
\text { realizado una } \\
\text { búsqueda de } \\
\text { información }\end{array}$ & $\begin{array}{l}\text { La búsqueda de } \\
\text { información ha } \\
\text { sido entusiasta, } \\
\text { pero no por parte } \\
\text { de todos }\end{array}$ & $\begin{array}{l}\text { Todos los } \\
\text { miembros del } \\
\text { grupo han } \\
\text { participado en la } \\
\text { recopilación de } \\
\text { información de } \\
\text { forma activa }\end{array}$ \\
\hline 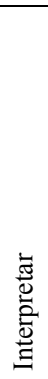 & $\begin{array}{l}\text { El grupo ha } \\
\text { interpretado } \\
\text { adecuadamente el } \\
\text { problema, } \\
\text { identificando sus } \\
\text { claves y } \\
\text { relacionando con } \\
\text { las mismas la } \\
\text { información } \\
\text { recopilada. }\end{array}$ & $\begin{array}{l}\text { No han podido } \\
\text { identificar las } \\
\text { claves del } \\
\text { problema ni } \\
\text { relacionarlas } \\
\text { con la } \\
\text { información } \\
\text { previa }\end{array}$ & $\begin{array}{l}\text { Han identificado } \\
\text { las claves } \\
\text { razonablemente } \\
\text { sin relacionarlas } \\
\text { con la } \\
\text { información }\end{array}$ & $\begin{array}{l}\text { Han identificado } \\
\text { las claves y } \\
\text { encontrado } \\
\text { algunas } \\
\text { relaciones con la } \\
\text { información de } \\
\text { partida }\end{array}$ & $\begin{array}{l}\text { La identificación } \\
\text { de claves es } \\
\text { adecuada y bien } \\
\text { relacionada con } \\
\text { la información }\end{array}$ \\
\hline 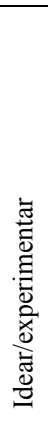 & $\begin{array}{l}\text { El grupo se ha } \\
\text { enfrentado al } \\
\text { problema con } \\
\text { creatividad, } \\
\text { proporcionando } \\
\text { ideas y enfoques } \\
\text { novedosos o poco } \\
\text { convencionales. } \\
\text { Han planteado } \\
\text { soluciones poco } \\
\text { exploradas }\end{array}$ & $\begin{array}{l}\text { No han } \\
\text { aportado idea } \\
\text { original o } \\
\text { novedosa para } \\
\text { encarar el } \\
\text { problema }\end{array}$ & $\begin{array}{l}\text { Aportan alguna } \\
\text { idea basada en } \\
\text { soluciones previas } \\
\text { convencionales }\end{array}$ & $\begin{array}{l}\text { Aportan alguna } \\
\text { idea original } \\
\text { buscando } \\
\text { soluciones } \\
\text { diferentes a las } \\
\text { existentes }\end{array}$ & $\begin{array}{l}\text { Han sido muy } \\
\text { creativos } \\
\text { generando ideas y } \\
\text { enfoques } \\
\text { originales }\end{array}$ \\
\hline
\end{tabular}

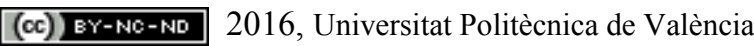

Congreso In-Red (2016) 


\begin{tabular}{|c|c|c|c|c|c|}
\hline \multirow{2}{*}{\multicolumn{2}{|c|}{ Epígrafe }} & \multicolumn{4}{|c|}{ Evaluación } \\
\hline & & 0 & 1 & 2 & 3 \\
\hline 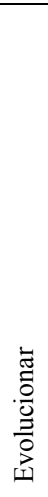 & $\begin{array}{l}\text { Tras las reuniones } \\
\text { de seguimiento y } \\
\text { en la propia } \\
\text { presentación del } \\
\text { trabajo, se } \\
\text { observa que las } \\
\text { propuestas } \\
\text { evolucionan en } \\
\text { función de los } \\
\text { comentarios y } \\
\text { reflexiones sobre } \\
\text { las primeras } \\
\text { versiones }\end{array}$ & $\begin{array}{l}\text { Las reuniones } \\
\text { de seguimiento } \\
\text { no parecen } \\
\text { haber influido } \\
\text { en el trabajo }\end{array}$ & $\begin{array}{l}\text { Ha habido } \\
\text { aportaciones en } \\
\text { las reuniones, } \\
\text { pero la línea del } \\
\text { trabajo apenas ha } \\
\text { cambiado }\end{array}$ & $\begin{array}{l}\text { Las reuniones de } \\
\text { grupo han hecho } \\
\text { evolucionar el } \\
\text { trabajo }\end{array}$ & $\begin{array}{l}\text { Las reuniones han } \\
\text { hecho evolucionar } \\
\text { el trabajo, y se } \\
\text { nota en la } \\
\text { presentación del } \\
\text { resultado }\end{array}$ \\
\hline 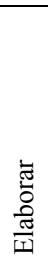 & $\begin{array}{l}\text { La presentación } \\
\text { del resultado } \\
\text { muestra una } \\
\text { maduración de las } \\
\text { ideas a lo largo } \\
\text { del trabajo } \\
\text { realizado. }\end{array}$ & $\begin{array}{l}\text { La solución } \\
\text { propuesta } \\
\text { apenas se } \\
\text { diferencia de la } \\
\text { idea inicial }\end{array}$ & $\begin{array}{l}\text { La solución } \\
\text { muestra algunas } \\
\text { ideas surgidas en } \\
\text { el desarrollo }\end{array}$ & $\begin{array}{l}\text { La solución es } \\
\text { madura, pero } \\
\text { poco creativa }\end{array}$ & $\begin{array}{l}\text { La solución es } \\
\text { reflejo de } \\
\text { creatividad y } \\
\text { maduración de } \\
\text { ideas a lo largo } \\
\text { del tiempo }\end{array}$ \\
\hline
\end{tabular}

\section{Resultados}

En los dos años de impartición de la asignatura, hemos observado como los equipos de alumnos presentaban trabajos de calidades dispares: desde trabajos serios y bien documentados tanto en lo que respecta a lo puramente técnico como a la implicación social de la respuesta, hasta trabajos realizados para cubrir el expediente y salir del paso en la asignatura. Resulta curioso analizar la evaluación que de estos trabajos hacen tanto profesores como alumnos. En ambos cursos impartidos, se organizaron cuatro equipos cada año. De esa muestra de ocho equipos realizando proyectos según dinámicas DT y su posterior evaluación podemos extraer algunas conclusiones:

1. En general, los compañeros suelen ser algo más laxos en sus puntuaciones que los profesores, con diferencias en torno a 2 puntos sobre 15 con respecto a las calificaciones proporcionadas por los docentes.

2. A pesar de esta mayor "benevolencia", los compañeros detectan diferencias entre los trabajos, esto es, no proporcionan la misma puntuación a todos los trabajos, lo que entendemos indica que las rúbricas tienen un efecto positivo, ya que ayudan a objetivizar la calificación propuesta.

3. Además, y esto lo consideramos muy resaltable, la coincidencia entre compañeros y profesores es total en cuanto a la clasificación de los trabajos evaluados: si organizamos los grupos por las puntuaciones obtenidas en cada uno de los dos cursos, siempre el mejor considerado por los profesores lo ha sido también por los compañeros. Y de la misma forma todos han sido situados en la misma posición en 
el supuesto ranking, incluyendo el considerado de peor calidad: en ambos años coincidieron en su apreciación profesores y alumnos.

Consideramos que es importante reconocer la forma coral en la que se realiza la evalución de los trabajos como una aproximación coherente a los objetivos y competencias de la asignatura y a la propia metodología DT. La nota obtenida por los trabajos no viene determinada por la evaluación que el profesor pueda realizar de las competencias técnicas demostradas por sus alumnos. Por el contrario, son todos los profesores de la asignatura y también el conjunto de compañeros, los que en base a la presentación y los informes generados por los alumnos determinan la calidad del trabajo realizado, teniendo en cuenta su propia experiencia en la propia asignatura y los criterios incluidos en las rúbricas. Esta forma de evaluación constituye un elemento básico en el desarrollo coherente de la asignatura en base a su condición multidisciplinar y socialmente transcendente, más allá de las tecnologías propias de las disciplinas ingenieriles.

En cuanto a la experiencia personal de los profesores, se puede decir que esta ha resultado enriquecedora. La situación de partida no era probablemente la mejor: profesores acostumbrados a impartir asignaturas de Ingeniería enfrentándose a una asignatura con un marcado carácter social: implicaciones éticas, la historia de las Telecomunicaciones, la implicación de la Ingeniería en la Sociedad, etc. A este contexto se añade que procedíamos de tres departamentos diferentes y no teníamos experiencia previa de impartir una asignatura juntos. Con el paso del tiempo, la experiencia está resultando muy positiva. Por un lado, desde puntos de vista diferentes, y en algunos casos divergentes, hemos conseguido construir una asignatura en la que los alumnos toman conciencia de su papel, como futuros ingenieros, en la sociedad que les rodea. Por otro lado, actuamos como guías en un proceso de descubrimiento de este papel, apoyando su maduración personal y acompañando con un método en el que por sí mismos se involucran en la realización de un proyecto grupal.

Por otra parte, en las encuestas que la Universidad propone anualmente a los alumnos para evaluar a profesores y asignaturas, los estudiantes puntúan con 4,11 sobre 5 a la asignatura, siendo la media del curso de 4,16 y la de la Universidad en su conjunto de 3,93. Analizando la valoración que hacen los alumnos de la propia asignatura, se comprueba que la consideran con un nivel de importancia similar a las otras de primer curso del Máster en Ingeniería de Telecomunicación, a pesar de tener unos contenidos mucho menos tecnológicos que aquellas con las que se compara. La puntuación sobre la coordinación entre los cuatro profesores que la imparten se valora con un 4,46 sobre 5, lo que interpretamos muy positivamente ya que indica que el procedimiento de evaluación por parte de los profesores que no intervienen en el seguimiento de los grupos se aprecia como adecuado, así como la conexión entre la docencia de aula y los proyectos en equipo.

Resultan peor valoradas las condiciones en las que se imparte la asignatura, un 3,91 sobre 5 , lo que interpretamos como una necesidad de mejorar las salas en las que se realizan las reuniones con los profesores, de modo que permitan una mejor interacción y un más ágil prototipado y desarrollo.

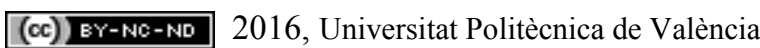

Congreso In-Red (2016) 


\section{Conclusiones}

A lo largo de este artículo hemos explicado nuestra experiencia en la aplicación de técnicas de Design Thinking para el desarrollo de proyectos creativos con aplicaciones directas en la sociedad en la que se desenvuelven los alumnos del Máster en Ingeniería de Telecomunicación de la Universidad de Vigo. La experiencia presentada tiene una limitación temporal de dos años, que son los que se lleva impartiendo el primer curso académico de dicha titulación, y un número de alumnos participantes relativamente pequeño: 36 entre los dos años. A pesar de estas limitaciones temporal y muestral, las tendencias que venimos observando se repiten, lo que nos lleva a suponer que la propuesta puede tener recorrido en la titulación y resultar de utilidad en la formación y posterior desarrollo profesional de nuestros alumnos y futuros titulados en Ingeniería de Telecomunicación.

Hemos explicado en qué consiste la experiencia, pero también detalles que consideramos importantes para la repetibilidad de la misma, como son el procedimiento de creación de grupos de trabajo, el desarrollo de la actividad y la evaluación:

- Para la creación de los grupos, se emplea un procedimiento similar a la selección de personal de una empresa, de modo que los alumnos se acostumbren a trabajar en equipo con compañeros seleccionados externamente, no con los amigos con los que suelen estudiar o trabajar. Esto supone un reto de madurez para nuestros futuros titulados.

- En cuanto al desarrollo de la actividad, se establecen diversos hitos presenciales con el profesor responsable, que ayudan a guiar el trabajo autónomo de los grupos. Se trata de ayudar a no perder el norte de la tarea que el grupo tiene entre manos, pero sin interferir directamente en lo que están desarrollando.

- En cuanto a cómo se evalúan los trabajos, se hace especial hincapié en la importancia de tener unas reglas de juego claras (y, por ello, se emplean rúbricas que se conocen antes de comenzar la tarea) así como la aportación de la evaluación combinada que, sobre el trabajo presentado por cada equipo, hacen los profesores de la asignatura y los demás alumnos (sus pares). El procedimiento de evaluación se considera de vital importancia en todo el proceso: no sólo permite a los profesores valorar los resultados de la actividad; también permite a los alumnos tomar conciencia de la calidad de las propuestas presentadas y contextualizarlas por comparación con las presentadas por los demás grupos. Se constata un alto grado de coherencia entre las calificaciones de los profesores y de los alumnos, quizá no tanto en la nota final como en la ordenación de los trabajos según la calificación otorgada. Entendemos que la responsabilidad de la evaluación aporta un incentivo a los alumnos, pero también acompaña en el proceso de maduración personal necesario para su futura incorporación al mercado laboral y a la vida adulta.

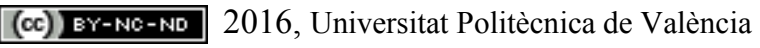


Finalmente, valoramos la actividad tanto objetiva como subjetivamente, a partir de resultados de encuestas de los alumnos y de las percepciones subjetivas de profesores, demostrando que los alumnos valoran positivamente este tipo de experiencias que contribuyen a incrementar sus competencias pero también, y muy efectivamente, su madurez personal y su implicación en la Sociedad en la que se desarrollan.

En los próximos cursos académicos, la intención del equipo docente de la asignatura es impulsar esta experiencia de inmersión en Design Thinking para el desarrollo de un pequeño proyecto, mejorando la planificación de la explicación del método y de las reuniones de seguimiento; revisando las rúbricas; buscando el modo de evaluar de forma individual dentro de cada grupo para evitar que algunos alumnos puedan sentir la tentación de dejarse llevar por el trabajo del resto de compañeros; y proporcionando mejores entornos de trabajo para el desarrollo de las actividades. Para esto último se cuenta con el apoyo de la Escuela, que recientemente ha adaptado un espacio como sala de desarrollo para proyectos colaborativos.

\section{Agradecimientos}

Este trabajo ha sido realizado en el marco del proyecto "Development of Innovative AcadeMy ON the basis of DT teaching (DiamonDT)", referencia 2015-1-PL01-KA203016844, financiado por la Unión Europea, programa Erasmus+ key action 2.

\section{Referencias}

CAIN, S. (2012). El poder de los introvertidos. RBA Libros.

CATMULL, E. (2015). Creatividad, S.A. Penguin Random House.

CAEIRO, M., CUIÑAS, I., FERNÁNDEZ-IGLESIAS, M., MARIÑO, P. (2015). La Ingeniería de Telecomunicación en la Sociedad de la Información, Guía Docente de la asignatura. $<$ https://seix.uvigo.es/docnetnuevo/guia_docent/index.php?centre $=305 \&$ ensenyament $=\mathrm{V} 05 \mathrm{M} 145 \mathrm{~V} 01 \&$ assignatura $=\mathrm{V} 05$ M145V01101\&idioma_assig=cast $>$ [Consulta 4 de abril de 2016]

España. Orden CIN/355/2009, de 9 de febrero, por la que se establecen los requisitos para la verificación de los títulos universitarios oficiales que habiliten para el ejercicio de la profesión de Ingeniero de Telecomunicación. Boletín Oficial del Estado, 20 febrero de 2009, núm. 44, p. 18165-18169.

IDEO (2012). Design Thinking for Educators Toolkit.

$<$ http://www.designthinkingforeducators.com/ > [Consulta 16 de marzo de 2016]

KAHNEMAN, D. (2011). Thinking, fast and slow. Penguin Books.

JOHANSSON-SKÖLDBERG, U., WOODILLA, J. y ÇETINKAYA, M. (2013). "Design Thinking: Past, Present and Possible Futures" en Creativity and Innovation Management, vol. 22, issue 2, p. 121-146. 
LIEDTKA, J. y OLGIVIE, T.(2011). Designing for growth; a design thinking tool kit for managers. Columbia University Press.

OWEN, C. (2007). "Design Thinking: Notes on its Nature and Use" en Design Research Quarterly, vol. 2, issue 1, p. 16-27. 